\title{
Reply: Effect of Divalproex Combined with Olanzapine or Risperidone in Patients with an Acute Exacerbation of Schizophrenia
}

\author{
Daniel E Casey*,1, David G Daniel ${ }^{2,3}$, Adel A Wassef ${ }^{4}$, Katherine A Tracy ${ }^{5,6}$, Patricia Wozniak ${ }^{5}$, \\ and Kenneth W Sommerville ${ }^{7}$ \\ 'Oregon Health \& Science University, Portland, OR, USA; ${ }^{2}$ George Washington University, Washington, DC, USA; ${ }^{3}$ Bioniche Development, Falls \\ Church, VA, USA; ${ }^{4}$ HCPC-University of Texas, Houston, TX, USA; ${ }^{5}$ Abbott Laboratories, Abbott Park, IL, USA; ${ }^{6}$ University of Illinois, Chicago, IL, \\ USA; ${ }^{7}$ Schwarz Biosciences, Research Triangle Park, NC, USA
}

Neuropsychopharmacology (2004) 29, 637-638. doi:I0.1038/sj.npp. 1300369

\section{Sir}

In their commentary on Casey et al, Boylan and Labovitz raise a number of important issues. The authors first propose that study groups 'were analyzed separately or in a combination in ways that enhanced the appearance of benefit and reduced the evidence of risk.' The efficacy analysis reported in the manuscript was the protocolspecified primary analysis. The study was powered to compare the efficacy between monotherapy (olanzapine or risperidone) and combination therapy (divalproex added to one of the two atypical antipsychotics). Two atypicals were chosen to increase the generalizability of the results, and olanzapine and risperidone were specifically chosen based on a studies showing similar efficacy (Davis et al, 2003). Boylan and Labovitz argue that combining antipsychotics in the efficacy analysis was justified on the basis of a test for interaction that was underpowered to detect a difference in a study of this size. The purpose of the test for an interaction in the primary efficacy outcome was to verify no serious departure from the assumption of no difference between antipsychotic groups. However, a visual inspection of the separate antipsychotic groups (Figure 1 in the original study) suggests that the separate antipsychotics were similar in their efficacy (combination therapy is on the favorable side of efficacy at each time point for both drugs by a similar magnitude).

Boylan and Labovitz criticize the separation of the two antipsychotic drugs for the purposes of safety analysis on the basis of reduced power. However, the separation of the

\footnotetext{
*Correspondence: DE Casey, 318I S.W. Sam Jackson Park Road, UHN80, Portland, OR 97239-3098, USA, Tel: + 503 418 1291,

E-mail: caseyd@ohsu.edu

Received 28 October 2003; accepted 06 November 2003

Online publication: 12 November 2003 at http://www.acnp.org/ citations/Npp I | | 20303496/default.pdf
}

two antipsychotics for the safety analysis was done based on the expected differences on the safety profiles for the two drugs (unlike the expected similarity on efficacy). Therefore, separate safety analyses for each drug were presented in the interest of full disclosure of safety data and a separate analysis of the combined safety data does not support a more adverse profile for the divalproex combination therapy even when the safety data are combined for the two antipsychotic drugs. Constipation, joint disorder, and pharyngitis were reported with statistically significantly higher incidence in the antipsychotic monotherapy group vs the combination group, while no adverse events occurred in a statistically significantly greater proportion of subjects in the combination group than in the antipsychotic monotherapy group.

With regard to the failure to find a benefit at Day 28, the authors charge that we did not entertain the possibility that the failure was a true negative and that the initial benefit was transient. Certainly, it must be acknowledged that the lack of statistical significance at Day 28 may have been a true negative finding and, in our paper, we state that it is not known whether the efficacy results observed will be sustained, increased, or diminished over longer periods of follow-up.' Further research is being conducted to address the failure at Day 28 and to investigate whether the efficacy effect is transient or will be maintained for a longer period of time.

The authors also suggest that the early effect of efficacy may have been due to nonspecific sedation. This assertion is not supported by the data. No significant difference in sedation was found with the addition of divalproex to either drug. Furthermore, an item analysis found improvements on core positive symptoms such as delusions, unusual thought content, and excitement. Also, drugs that induce sedation (such as lorazepam) in psychotic patients are not effective treatments for positive symptoms. There was a higher incidence of somnolence as an adverse event for the 
combination therapy group than for the monotherapy group (34 vs 23\%), but this difference was not statistically significant $(p=0.069$ from Fisher's exact test, $p=0.062$ from a $\chi^{2}$ test). To help answer the question of whether the positive effect of divalproex was a result of increased somnolence, we performed an ANOVA for PANSS. Total change from baseline scores with the following factors: treatment (mono vs combo), somnolence as an adverse event during the study (present, absent), and the interaction of treatment and somnolence. Treatment differences were similar for those who reported somnolence during the study and those who did not, with statistically significant treatment effects favoring combination therapy at Days 7 , 10 , and 14 , and a trend $(p<0.10)$ favoring combination therapy on Days 3 and 5. There was no evidence of an interaction.
Finally, we respectfully disagree with the conclusion of the authors that the lack of significance on Day 28 weakens the support for the use of divalproex in schizophrenia. The ability to have early (beginning at Day 3 ) benefit on positive symptoms in acute exacerbations of schizophrenia contributes to the acute management and stabilization of these patients, and is particularly important in a time of short hospital stays.

Sincerely,

\section{REFERENCES}

Davis JM, Chen N, Glick ID (2003). A meta-analysis of the efficacy of second-generation antipsychotics. Arch General Psychiatry 60: 553-564. 